ACCEPTED MANUSCRIPT

\title{
Surface energy patterning for ink-independent process optimization of inkjet-printed electronics
}

To cite this article before publication: Stefan Schlisske et al 2020 Flex. Print. Electron. in press https://doi.org/10.1088/2058-8585/abcc79

\section{Manuscript version: Accepted Manuscript}

Accepted Manuscript is "the version of the article accepted for publication including all changes made as a result of the peer review process, and which may also include the addition to the article by IOP Publishing of a header, an article ID, a cover sheet and/or an 'Accepted

Manuscript' watermark, but excluding any other editing, typesetting or other changes made by IOP Publishing and/or its licensors"

This Accepted Manuscript is @ 2020 IOP Publishing Ltd.

During the embargo period (the 12 month period from the publication of the Version of Record of this article), the Accepted Manuscript is fully protected by copyright and cannot be reused or reposted elsewhere.

As the Version of Record of this article is going to be / has been published on a subscription basis, this Accepted Manuscript is available for reuse under a CC BY-NC-ND 3.0 licence after the 12 month embargo period.

After the embargo period, everyone is permitted to use copy and redistribute this article for non-commercial purposes only, provided that they adhere to all the terms of the licence https://creativecommons.org/licences/by-nc-nd/3.0

Although reasonable endeavours have been taken to obtain all necessary permissions from third parties to include their copyrighted content within this article, their full citation and copyright line may not be present in this Accepted Manuscript version. Before using any content from this article, please refer to the Version of Record on IOPscience once published for full citation and copyright details, as permissions will likely be required. All third party content is fully copyright protected, unless specifically stated otherwise in the figure caption in the Version of Record.

View the article online for updates and enhancements. 


\title{
Surface energy patterning for ink-independent process optimization of inkjet-printed electronics
}

\author{
Stefan Schlisske ${ }^{1,2}$, Sebastian Raths ${ }^{3}$, Luis A. Ruiz-Preciado ${ }^{1,2}$, Uli Lemmer $^{1,4}$, \\ Kai Exner ${ }^{5}$ and Gerardo Hernandez-Sosa ${ }^{1,2, *}$ \\ ${ }^{1}$ Light Technology Institute, Karlsruhe Institute of Technology, Engesserstrasse 13, 76131 Karlsruhe, \\ Germany \\ ${ }^{2}$ InnovationLab, Speyerer Strasse 4, 69115 Heidelberg, Germany \\ ${ }^{3}$ BASF SE, Carl-Bosch-Strasse 38, 67056 Ludwigshafen, Germany \\ ${ }^{4}$ Institute of Microstructure Technology, Karlsruhe Institute of Technology, \\ Hermann-von-Helmholtz Platz 1, 76344 Eggenstein-Leopoldshafen, Germany \\ ${ }^{5}$ BASF New Business GmbH, Benckiserplatz 1, 67059 Ludwigshafen, Germany \\ E-mail: gerardo.sosa@kit.edu \\ Received xxxxxx \\ Accepted for publication $\mathrm{xxxxxx}$ \\ Published xxxxxx \\ Abstract
}

The applicability of inkjet-printed (opto-)electrical devices are hindered by their low lateral resolution, when compared to conventional techniques. The low lateral resolution is mainly caused by the flow and spreading of the functionalink on the substrate, which is determined by the substrate-ink-interaction. Recent apporaches, that confine and controll the spreading have been developed. However, they suffer from low lateral resolution or the usage of physical barriers. The later needs an adjustment of the ink or may contain an overlaid height information. Both cases are not always applicable when fabriacting functional devices. Herein we report the utilization of a surface energy patterning approach based on siloxane self-assembled monolayers. The obtained energetic differences control the flow and suppress the spreading of the ink, without creating the necessity to alter the ink composition. Furthermore this approach leads to an improved structural fidelity and printing resolution of arbitrary shapes. With that, we were able to print silver- and gold-electrodes for organic filedeffect transistors with a channel length of $<25 \mu \mathrm{m}$, fabricating feature sizes below the footprint of a single drop. The electrical characterization of these transistors revealed that the utilization of this surface energy patterning has no negative influence on the device performance.

The introduced approach facilitates the process development and improves the quality and resolution of printed features. This will facilitate the fabrication of high-quality and high resolution printed electronic devices.

Keywords: wetting, dewetting, two-dimensional patterning, functional printing, organic field-effect transistors 


\section{Introduction}

Inkjet printing has emerged as one of the most promising methods for the future cost-efficient fabrication of (opto-) electronic devices. ${ }^{[1,2]}$ The digital and additive processing capabilities enable a high degree of personalization alongside with low material consumption, essential for new technologies in the areas of "Internet-of-Things", healthcare, wearables, and communication. ${ }^{[3-7]}$ Numerous recent studies report on the successful fabrication and systematic integration of electronic components such as organic light-emitting diodes, photodiodes, photovoltaics or integrated circuits through inkjet printing. ${ }^{[8-12]}$ Nevertheless, the further advancement of these printed electronic devices is currently being hindered, by the lower lateral resolution of the inkjet printing process compared to conventionally used fabrication techniques such as lithography or vacuum-evaporation. To understand the underlying reason for this crucial limitation one should consider the formation, control and behavior of a single droplet of ink being ejected from the print head and being deposited on a substrate. ${ }^{[13,14]}$ The highest achievable resolution corresponds to the smallest possible footprint of a droplet on the substrate, which in return is dictated by its volume and the contact angle to the substrate. ${ }^{[10,15]}$ The former is limited by the print-head characteristics and reaches a minimum of $2 \mathrm{pL}$ for state-of-the-art industrial print heads. ${ }^{[16]}$ The latter mainly depends on the substrate-ink interaction defined by the surface tension and surface free energy (SFE). However, this interaction or wetting behavior is specific to a particular combination of substrate and ink and cannot always be transferred to other material systems. ${ }^{[17,18]}$

Self-assembled monolayers $(\mathrm{SAMs})^{[19-21]}$ and plasma treatments ${ }^{[22,23]}$ have typically been used to control the SFE of the substrate and increase the transferability of inkjet printing processes. The purpose of these approaches is to alter the surface conditions of the whole substrate to adjust the feature size of the printed patterns. Although this isotropic surface treatment allows for reproducible surface conditions independently of the substrate, the printing resolution is still coupled to the physical processes between the "new" substrate surface and the ink. ${ }^{[17]}$ Additionally, a common problem is, that by confining the spreading of ink by lowering the surface energy the adhesion of the structures is reduced. ${ }^{[24]}$ This has promoted the development of substrate modification processes that overlay lateral information to further decrease the resolution by local SFE contrast such âs the utilization of physical barriers ${ }^{[25-28]}$ or so-called dewetting structures ${ }^{[29-31]}$. Using such structures results in an anisotropic landscape that limits or guides the spreading of the deposited ink via hydrophilic and hydrophobic areas or through capillary effects. ${ }^{[32]}$ However, these physical barriers can reach heights in the range of several micrometers, which can add complexity to the fabrication of multilayer devices. Alternatively, the structuring of SAMs has been discussed in literature to obtain 2D-dewetting patterns without such an overlaid height information. These approaches have been recently used to assist the fabrication of solution processed organic field effect transistors (OFET). ${ }^{[29,30,33]}$ In these examples, the ink was preferentially driven towards areas with a suitable SFE and was repelled elsewhere, thus adding 2D-patterning capabilities to the used coating processes. The drawback of this approach resides in the formation of defects when the desired structures are too close to each other or the accumulation of ink residues when they are too far apart. ${ }^{[30]}$ Both, the application of physical barriers and 2D-dewetting patterns require the adjustment of the deposition process, ink composition, or pattern design to optimize printing resolution. In this context, a common optimization process is the modification of the inks by adding high surface-tension solvents to promote the dewetting. ${ }^{[30]}$ However, this step is not fully applicable when using commercially available inkjet inks, since changing its formulation might alter the stability of the drop formation or the material functionality in the dried film. ${ }^{[34,35]}$

In this work, we combine the patterning of SAMs with the precision of inkjet printing to facilitate the fabrication and to improve the accuracy and resolution of printed structures. The developed process helps to actively suppress the spreading of a commercially available nanoparticle-based silver inkjet ink and with that the fabrication of high quality, sub-resolution features independently from the deposition direction and in a single step printing process. In our approach, the ink is printed on areas with high surface energy on which the inks exhibit small contact angle and therefore a good adhesion can be expected. The ink is confined by the low surface energy around the printed structure and is not deposited on the low surface energy areas. The investigation of the adhesion of the inks is outside of the scope of this work. Additionally, we could show the flexibility of this approach by using two inks based on two different solvent systems, ether- vs. water-based, without the need to adjust the pre-structuring process or deposition parameters. With that, we were able to fabricate source-drain electrodes for fully solution-processed OFETs and could show that the additional pre-structuring steps have no negative influence on the device performance.

\section{Materials \& Methods}

\subsection{Fabrication and characterization of dewetting structures}

The influence of the surface tension enhanced resolution improvement was examined on borofloat 33 glass slides (Schott, Germany). Unless otherwise mentioned, all fabrication steps were performed at room temperature in ambient conditions under clean room conditions. The glass 
substrates were cleaned in an ultrasonic bath for 10 min with acetone and isopropyl alcohol and dried with a nitrogen gun after each step and were exposed to oxygen plasma for 5 min (Tetra 30, Diener Electronics $\mathrm{GmbH}+\mathrm{Co} . \mathrm{KG}$ ).

To increase the hydrophobicity and lower the surface free energy (SFE) the glass substrates were treated with a 1 vol.\% solution of $1 \mathrm{H}, 1 \mathrm{H}, 2 \mathrm{H}, 2 \mathrm{H}$-perfluorodecyltriethoxysilane (PFDTS) (AlfaAesar 97\%), triethoxyoctylsilane (TEOS) (Sigma-Aldrich 98\%), and trimethoxy-(3,3,3trifluoropropyl)-silane (TTFPS) (Alfa Aesar 97\%) in ethanol. The solutions were spin-coated on the glass substrates for 45 $\mathrm{s}$ at $1500 \mathrm{rpm}$ and an initial acceleration of $500 \mathrm{rpm} / \mathrm{s}$. After the deposition the substrates were annealed on a hotplate at 95 ${ }^{\circ} \mathrm{C}$ for $5 \mathrm{~min}$, rinsed with isopropyl alcohol and dried with nitrogen. The successful SAM removal was examined with

Static contact angle measurement (DSA 100, Krüss) of droplets with a nominal volume of $0.75 \mu$ l of deionized water, diiodomethane, and ethylene glycol and the method of Owens-Wendt- Rabel-Kaelble (OWRK) as described according to previous work of Schlisske et al. ${ }^{[21]}$ For the contact angle determination, at least three droplets of each solution were deposited at different spots on a substrate. From each droplet at least 30 frames were analyzed and the contact angle was extracted with the help of a software (ADVANCE Krüss).The siloxanes were finally structured with argon (Ar-) plasma through different masks for different exposure times $(5-60 \mathrm{~s})$. We used laser cut stainless steel masks (Becktronic $\mathrm{GmbH}$ ) and polyimide-foil masks (CADiLAC Laser $\mathrm{GmbH}$ ). Best results were obtained with $20 \mathrm{~s}$ exposure time. To determine the surface morphology we performed atomic force microscopy (AFM) anaylis with a DME DS 95 DualScope AFM under ambient conditions in tapping mode with a highly doped Si-cantilever (NanoWorld Arrow NCR) with a tip radius $<10 \mathrm{~nm}$ and a a resonance frequency of around $285 \mathrm{kHz}$.

\subsection{Fabrication and characterization of printed}

\section{structures}

Triethylene glycol monomethyl ether-based silver ink (SigmaAldrich Silverjet DGP 40LT 15C) was deposited via blade coating with different deposition parameters and inkjet printing. The water-based gold ink (C-Ink DryCure Au-J 1010B) was only deposited via inkjet printing. For inkjet printing we used a PixDro LP50 (MeyerBurger) equipped with a Dimatix DMC $10 \mathrm{pL}$ Cartridge and a custom designed waveform. The printhead temperature was set to $30^{\circ} \mathrm{C}$ and the substrate temperature was kept at $25^{\circ} \mathrm{C}$. The substrates were aligned with the onboard software of the printer using alignment markers consisting of the same dewetting structures as the other structures. These fiducials were made visible with a felt pen.

After deposition, the substrates were annealed at $120{ }^{\circ} \mathrm{C}$ for $10 \mathrm{~min}$ in ambient conditions. The structures were then analyzed with a Nikon Eclipse 80i optical microscope, which is equipped with a 5.0 megapixel camera. The length and width of the structures was then determined by image analysis with Nikon Basic Research software. For the geometrical characterization, the structures were analyzed at least at seven different spots. The values are presented with their respective mean deviation.

\subsection{Transistor fabrication and characterization}

For the fabrication of the transistors XPRD01B06 (BASF $\mathrm{SE}$ ) was used as semiconductor which was dissolved at a concentration of 0.75 percent by weight in a mixture of mesitylene, o-xylene and tetraline (1:1:2) and deposited by inkjet printing using a Fujifilm Dimatix DMP 2831 equipped with a Dimatix DMC 16110 cartridge resulting in a layer thickness of $50 \mathrm{~nm}$. The OSC is a Poly(diketopyrrolopyrroleterthiophne)-type material and was prepared according to Bijleveld et al. ${ }^{[36]}$ and Shaw et al. ${ }^{[37,38]}$ XDRD41L01 (BASF SE) dissolved at a concentration of 6 percent by weight in butyl acetate and was deposited by spincoating resulting in a layer thickness of $500 \mathrm{~nm}$. The dielectric is a polystyrenebased material, which is a proprietary material of BASF. The gate electrodes were evaporated from gold with a layer thickness of $50 \mathrm{~nm}$.

For the output characterisitcs we swept the drain voltage $\mathrm{V}_{\mathrm{D}}$ from +5 to $-30 \mathrm{~V}$ at constant gate voltages $\mathrm{V}_{\mathrm{G}}$ of +5 to -30 $\mathrm{V}$ in steps of $7 \mathrm{~V}$. The transfer characteristics were measured at constant drain voltages $V_{D}$ of -5 and $-30 \mathrm{~V}$ by sweeping the gate voltáge $\mathrm{V}_{\mathrm{G}}$ from +30 to $-30 \mathrm{~V}$.

\section{Results \& Discussion}

\subsection{Requirements for dewetting structures}

To obtain dewetting structures on a given substrate, a SFE contrast should be imposed onto two adjacent areas so that an ink preferably wets one of them. Such a favoured wetting on a specific surface area is typically observed if the contact angle of the ink to the substrate shows a difference $>30^{\circ}$ between the two areas. ${ }^{[26,31,33]}$ The investigated nanoparticle-based Agand $\mathrm{Au}$-inks have been designed for inkjet printing. The Ag ink exhibits a total surface tension of $35.5 \mathrm{mN} \mathrm{m}^{-1}$ with a polar contribution of $6.8 \mathrm{mN} \mathrm{m}^{-1}$ and a dispersive contribution of $28.7 \mathrm{mN} \mathrm{m}^{-1}{ }^{[26]}$ The surface tension of the Au-ink was measured to be $32.6 \mathrm{mN} \mathrm{m}^{-1}$ with a dispersive contribution of $28.4 \mathrm{mN} \mathrm{m}^{-1}$ and a polar contribution of $4.2 \mathrm{mN} \mathrm{m}^{-1}$. Due to the higher dispersive contributions, both inks should show good wettability on low-SFE polymeric substrates and worse wetting on polar substrates such as glass. ${ }^{[39,40]}$ Therefore, when depositing them onto glass substrates, the SFE of the repellent area should be tuned towards a low surface free energy with small dispersive and polar contributions to achieve the necessary contact angle difference between the 
Table 1: SFE of SAMs and contact angle of silver and gold ink

\begin{tabular}{|c|c|c|c|c|c|c|c|c|}
\hline \multirow{2}{*}{ SAM } & \multicolumn{2}{|c|}{ SFE [ $\mathbf{m N ~ m}^{-1}$ ] } & \multicolumn{2}{c|}{ Contact angle silver-ink [ ${ }^{\circ}$ ] } & \multicolumn{3}{c|}{ Contact angle gold-ink [ $\left.{ }^{\circ}\right]^{-1}$} \\
\cline { 2 - 9 } & dispersive & polar & on SAM & + Ar-plasma & $\Delta$ CA & on SAM & + Ar-plasma & $\Delta$ CA \\
\hline TTFPS & $19.5 \pm 0.1$ & $5.3 \pm 0.1$ & $35.4 \pm 0.3$ & $13.6 \pm 0.2$ & 21.8 & $49.4 \pm 0.2$ & $<10$ & 39.4 \\
\hline TEOS & $17.1 \pm 0.4$ & $6.4 \pm 0.3$ & $43.2 \pm 0.1$ & $13.9 \pm 0.4$ & 29.3 & $57.9 \pm 0.1$ & $<10$ & 47.9 \\
\hline PFDTS & $10.6 \pm 0.2$ & $2.7 \pm 0.2$ & $63.7 \pm 0.2$ & $18.9 \pm 0.4$ & 44.8 & $71.3 \pm 0.2$ & $10.0 \pm 0.89$ & 61.3 \\
\hline
\end{tabular}

on siloxane SAM before and after Ar-plasma treatment

different areas. In order to find the optimal substrate treatment for this purposed we compared the behavior of the inks onto glass covered with three different siloxane-based SAMs: trimethoxy-(3,3,3 trifluoropropyl)-silane (TTFPS), triethoxyoctylsilane (TEOS) and, $1 \mathrm{H}, 1 \mathrm{H}, 2 \mathrm{H}, 2 \mathrm{H}-$ perfluorodecyltriethoxysilane (PFDTS). These materials were selected due to their different functional groups resulting in total SFE of $24.8 \pm 0.2,23.5 \pm 0.7$, and $13.4 \pm 0.4 \mathrm{mN} \mathrm{m}^{-1}$ respectively. ${ }^{[21]}$ The corresponding polar $\left(\sigma_{\mathrm{p}}\right)$ and dispersive $\left(\sigma_{d}\right)$ components of the SFE can be found in table 1 .

To estimate the wetting behavior of the ink, we compared its surface tension (ST) to the calculated the wetting envelopes (WE) of the three different glass/SAM substrates. Such WE can be calculated with help of the OWRK-model which is based on Young's equation. ${ }^{[39-41]}$ The WE presented in figure 1a describe the polar and dispersive components of an ink that would yield a contact angle of $0^{\circ}$ relative to the substrate surface. Qualitatively, if the ST of an ink is located inside a WE, good wetting can be expected. In contrast, if the ink is located outside the WE, it will form a distinct contact angle: the further outside, the bigger the contact angle will be.

From figure 1a, it is clearly visible that both inks are located outside the WE of the SAM treated substrates, so that the formation of a distinct contact angle can be expected. To obtain a lateral structuring of the energetic landscape, we investigated the removal of the SAMs via exposure to plasma as commonly done in literature. ${ }^{[30,33]} \mathrm{We}$ exposed the SAM treated glass substrates to Ar-plasma through conventional shadow masks. As it can be seen in figure $1 \mathrm{~b}$ the contact angle significantly decreases for both the $\mathrm{Ag}_{-}$and $\mathrm{Au}_{-}$-ink after the PFDTS treated substrate was exposed to Ar-plasma. The optimization of the removal process is shown in the supporting information where an Ar-plasma equal or above $15 \mathrm{~s}$ was found to keep contact angle of water constantly below $10^{\circ}$. This reduction of the contact angle is caused by a significant increase of the polar contribution of the SFE after the removal of the SAM with AR-plasma as depicted by WE in figure 1a.

The resulting contact angles for the two inks on the three utilized SAMs, and after their removal with Ar-plasma, are depicted in table 1. For the pristine SAMs the contact angles increase with decreasing SFE reaching a maximum of $63.7^{\circ}$ and $71.4^{\circ}$ for the $\mathrm{Ag}$ - and Au-ink respectively, on the PFDTS treated substrates. In all cases, the contact angles drop down below $20^{\circ}$ after the substrate was exposed to Ar-plasma resulting in a contact angle difference higher than $30^{\circ}$, except for the silver ink on TTFPS-treated substrates, which led to a contact angle difference of only $21.8^{\circ}$
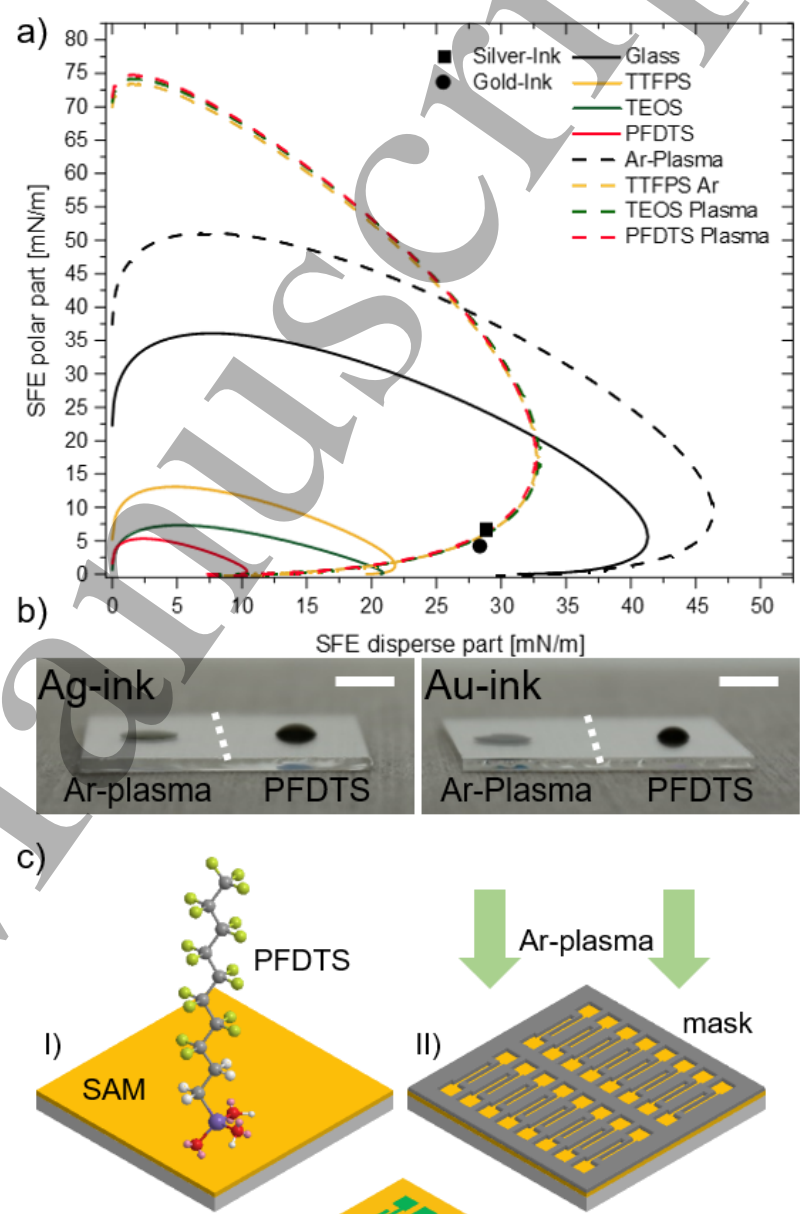

III)

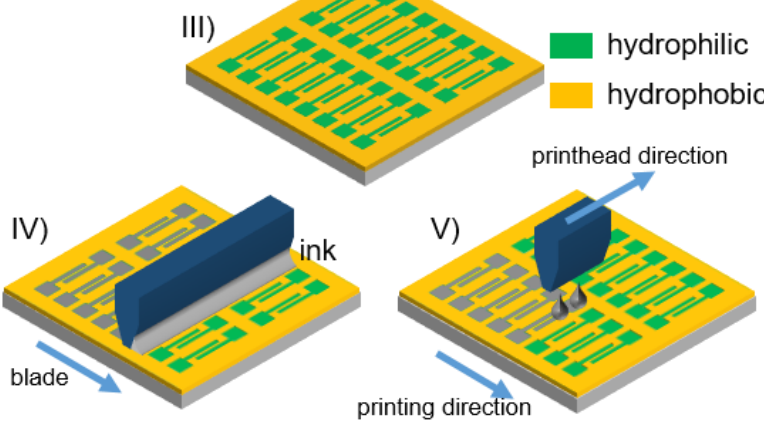

Figure 1: a) Wetting envelops of untreated glass (black), and SAM treated glass TTFPS (yellow), TEOS (green) and PFDTS (red) before (solid line) and after removal with Ar-plasma (dashed line). b) Photograph of the formation of contact angle of silver ink (left) and gold ink (right) on with Ar-plasma structured PFDTS treated glass (white bars represent $5 \mathrm{~mm}$ ). c) Schematic process of the 2D-SAM structuring for the fabrication of electrode structures. 
These results show that exposing the SAM treated substrates to Ar-plasma would allow the fabrication of areas with substantially different SFE, and thus enable the fabrication of 2D dewetting patterns. With that, we continued to investigate the influence of the deposition technique on the dewetting according to the schematic in figure 1c. For all further experiments, we used PFDTS-treated substrates as it shows the largest $\mathrm{CA}$ contrast for both the Ag- and the Auinks.

\subsection{Ink deposition via blade coating}

Electrode structures for thin-film electronics are typically fabricated via lithography or thermal evaporation. These techniques require either multiple steps or elaborated vacuum setups and are characterized by their high material consumption. Combining solution based coating techniques with SFE structured substrates is an alternative method that also allows the fabrication of 2D-patterns. We therefore investigated the applicability of our approach for the fabrication of interdigitated electrode structures via the doctor-blading technique utilizing a commercial available Agink. Interdigitated structures are of particular interest in printed electronics to increase the channel length of fieldeffect-transistors and to achieve a higher integration density. To optimize the deposition and dewetting quality of the structures we used the so-called Capillary Number $(\mathrm{Ca})$. This dimensionless number is calculated according to equation 1 with the viscosity $(\eta)$, the deposition speed (v) and the surface tension $(\sigma)$. Ca describes the tendency of a liquid to form the smallest possible surface area due to the surface tension, which is damped by the viscosity of the ink. In general an ink with a low $\mathrm{Ca}$ is needed to yield good dewetting, so that the viscosity does not suppress the dewetting process. ${ }^{[30,42]}$ This can be achieved by altering the ink composition, e.g. increasing the surface tension, or changing the deposition parameters. In our case, we solely optimized the deposition parameters not to compromise the stability and functionality of the ink.

$$
C a=\frac{\eta \cdot v}{\sigma}
$$

We tested different deposition speeds and blade gaps. The best results were achieved with the lowest blade speed of 1 $\mathrm{mm} \mathrm{s}^{-1}$ and therefore the lowest $\mathrm{Ca}=0.00043$. As it can be seen in figure $2 \mathrm{a}$, interdigitated structures with line widths of $59.3 \pm 3.7 \mu \mathrm{m}$ separated by $100 \mu \mathrm{m}$ gaps can be formed by surface tension driven self-alignment of the Ag ink. Their structural fidelity is independent of the orientation with respect to the coating direction, which is displayed by the white arrow. As it can be seen in Figure S2 in the supporting information, the surface topography remains almost unchanged. The PFDTS surface exhibits a roughness of $1.7 \mathrm{~nm}$ whereas after the Ar-plasma treatment the roughness was reduced to $0.7 \mathrm{~nm}$. With that it can be said that the spreading of the ink is confined solely due to energetic

barriers. Despite the low capillary number, it can be seen that not all the structures are formed and interconnected lines as well as randomly distributed stains are present on the substrate. These artefacts are highlighted with red circles in Figure 2 b,c,e. When decreasing the gap size $<50 \mu \mathrm{m}$ (see figure $2 \mathrm{~d}, \mathrm{e}$ ) we observed that in some cases these instabilities prevent the formation of well-defined electrodes.

The appearance of stains can be attributed to non-optimized structures, as the ink has to dewet from $>80 \%$ of the area (see the template depicted in figure S1 of the SI). Additionally, the low surface tension of the silver ink, which is lower than the water based inks in comparable studies from literature, ${ }^{[25,28,30]}$ also suppresses the formation of well-defined electrodes. The artefacts certainly would have a negative influence on the functionality and device performance so that their formation should be avoided. For instance, Pierre et al. ${ }^{[30]}$ optimized the yield of their structures by adding a high surface tension solvent additive to the ink and therefore decreasing $\mathrm{Ca}$. However, altering the ink composition is not always applicable, as this might have an influence on the ink stability or functionality of the dried film. As the ink is optimized for inkjet applications, we continued to investigate the deposition of the ink via inkjet printing only in the hydrophilic areas to suppress the bridging or stain formation.
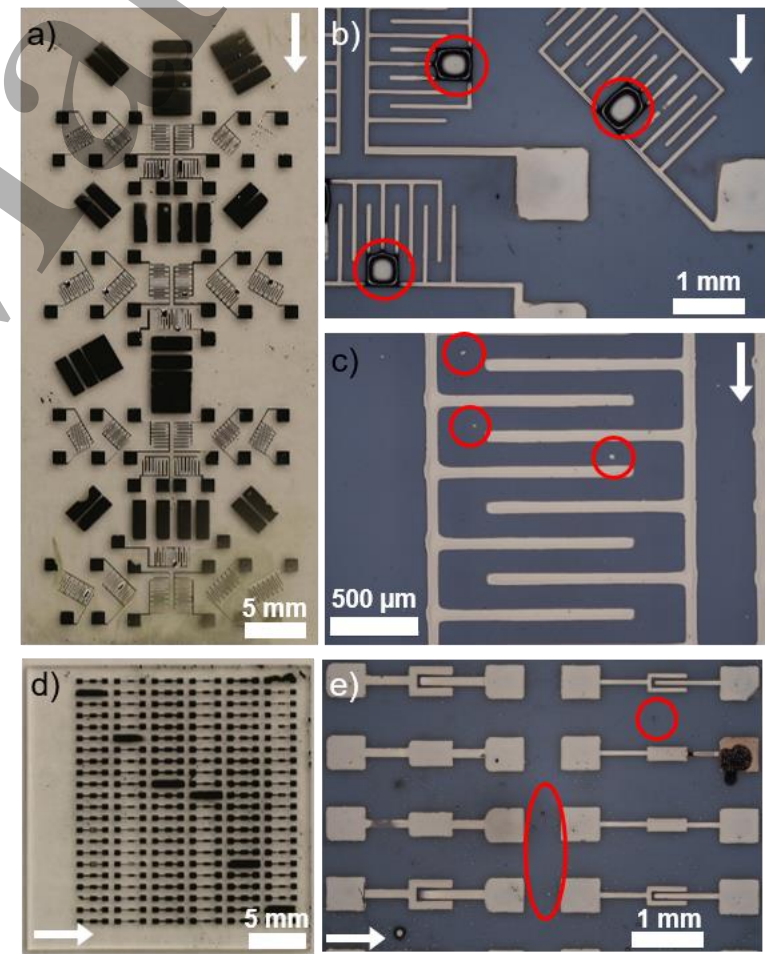

Figure 2: Photographs of silver ink deposited via blade coating for the formation of interdigitated electrode structures: a) The structural integrity is independent of the orientation, b) Interconnected lines and c) randomly appearing stains highlighted with red circles. d) Representation of the design flexibility and minimization of the feature sizes, e) Interconnected lines and stains on the substrate. White arrows indicate deposition direction. 


\subsection{Inkjet printing on pre-structured substrates}

Despite the digital nature of inkjet printing, which offers the possibility to deposit structures with freedom of design, inkjet printing suffers from low resolution compared to other printing techniques. ${ }^{[26,43]}$ Although technically drops could be placed $20 \mu \mathrm{m}$ apart, ${ }^{[44]}$ the spreading of the ink spoils this accuracy. ${ }^{[45]}$ Droplets deposited too close to each other can merge and therefore cause defects in the printed features. By utilizing the substrate pre-structuring, we investigate if the spreading of the ink can be actively suppressed compared to a non-structured surface treatment and whether this approach can be used to further increase the resolution.

For this, we transferred the technical drawing of the shadow mask used to define the SFE contrast areas into a printing pattern. Then we inkjet printed the metal inks only inside the hydrophilic areas where we previously removed the SAM. On the one hand, this local drop deposition would exclude the formation of defect and residual ink happening in a coating process. On the other hand, we ensure that the resolution of the printed lines is constrained to the size of the SFE contrast areas.

Table 2: Comparison of the line widths of inkjet printed silver and gold electrodes on structured and unstructured substrates.

\begin{tabular}{|c|c|c|c|c|}
\hline & \multicolumn{3}{|c|}{ Linewidth $[\mu \mathrm{m}]$} & \multirow{2}{*}{$\begin{array}{c}\text { Mask } \\
\text { opening } \\
{[\mu \mathrm{m}]}\end{array}$} \\
\hline Ink & $\begin{array}{c}\text { PFDTS + } \\
\text { Ar-plasma }\end{array}$ & $\begin{array}{c}\text { Glass + Ar- } \\
\text { plasma }\end{array}$ & Neat & \\
\hline $\mathrm{Ag}$ & $53.2 \pm 0.63$ & $89.32 \pm 0.53$ & $115.73 \pm 19.21$ & \multirow{2}{*}{$50.99 \pm 0.95$} \\
\hline $\mathrm{Au}$ & $50.90 \pm 1.37$ & $181.53 \pm 2.98$ & $87.61 \pm 4.85$ & \\
\hline
\end{tabular}

As it can be seen in figure $3 a$ the benefit of a pre-structured substrate is clearly visible. The opening in the evaporation mask was measured to be $50.99 \pm 0.95 \mu \mathrm{m}$ and could be replicated by pre-structuring the SAM and printing the silver ink onto the high SFE areas resulting in line widths of $53.2 \pm 0.63 \mu \mathrm{m}$ (highlighted with a green arrow in figure $3 \mathrm{a}$ ). The results for the water-based $\mathrm{Au}$-ink are similar, achieving line widths down to $50.9 \pm 1.37 \mu \mathrm{m}$. On the plasma treated areas, the single drop diameter of the Ag-ink is $64.39 \pm 0.85$ $\mu \mathrm{m}$. and $67.17 \pm 1.07 \mu \mathrm{m}$ for the Au-ink (see figure S5 in the supporting information). In both cases, the single drop diameter is higher than the obtained line width (figure $3 \mathrm{a}$ ), as the SAM confines the ink inside the narrow high surface energy areas fabricated by the plasma treatment, so that a subresolution printing is possible. Compared to bare glass, the line width of printed structures is decreased by $117 \%$ for the printed silver and by $72 \%$ for the printed gold lines. For the case of structures printed on Ar-plasma treated glass the linewidth was decreased by $67 \%$ in case of silver and $256 \%$ in case of gold. A comparison of the linewidths is presented in table 2. It can be observed that for the printed lines on the structured SAM substrates, the line widths variation along the electrode deviate by only $1.2 \%$ and $2.7 \%$ for the Ag- and Auink respectively. In the other cases, the line widths deviate by up to $17 \%$. This deviation is especially prominent at the end of the lines resp. electrodes where so-called bulging occurs. ${ }^{[46]}$ Thus, these results show that ink spreading and bulging could be effectively suppressed by using the pre-structuring and therefore the electrical field distribution between the two electrodes is expected to be improved.
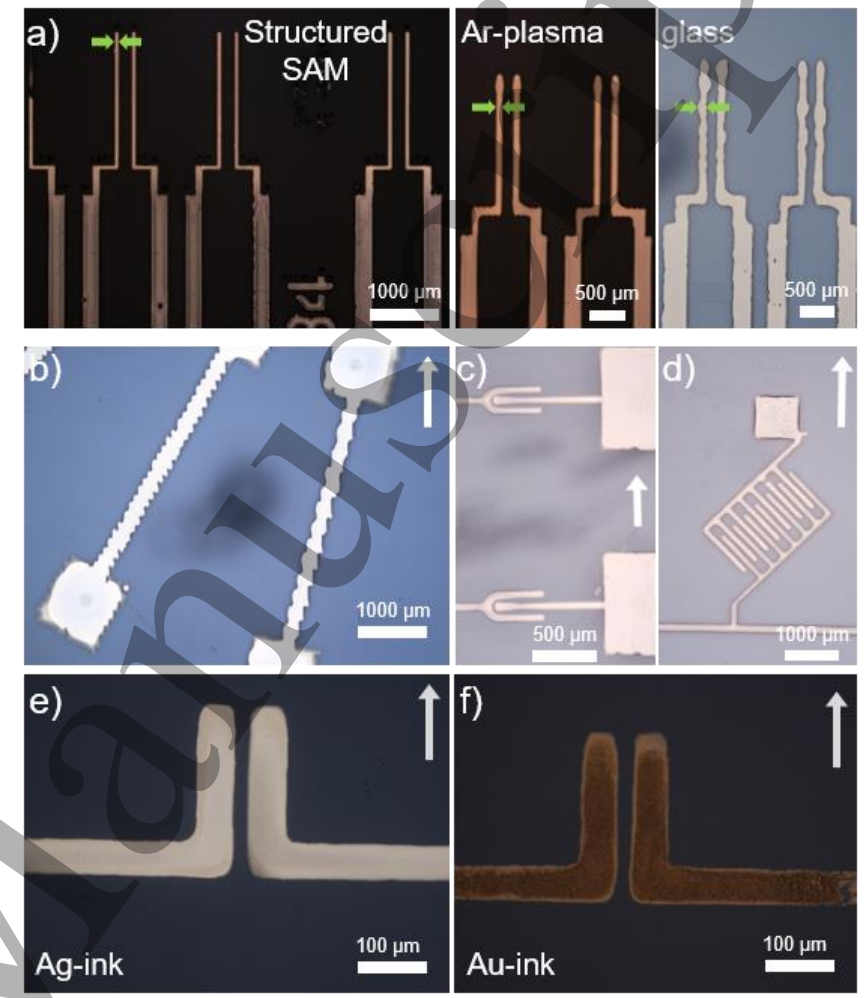

Figure 3: Microscopic images of a) inkjet printed silver electrodes on structured (PFDTS) and unstructured substrates; b) photograph of a not optimized structure on non-patterned glass substrate leading to saw-tooth like edges. $c, d)$ photographs of arbitrary shapes not optimized for inkjet printing on SAM structured substrates. Minimizing the feature size of e) silver and f) gold electrodes separated by $<25 \mu \mathrm{m}$. The arrows indicate printing direction.

As depicted in figure $3 \mathrm{~b}$-d the printing of arbitrary shapes also benefits from this approach. Structures, which are not oriented perpendicular or parallel to the printing direction, are typically hard to process by inkjet printing since these features cannot be translated correctly into a print pattern. As the generated pattern is based on a rectangular resolution grid, such structures can therefore exhibit saw-tooth like edges (see

figure $3 b$ and S3 in the SI). The print pattern generation is described in more detail in the supporting information. As depicted in figure $3 \mathrm{c}$, the patterned SAM approach produces sharp boundaries and a path for the ink to flow into, resulting in smooth and well-defined contours and edges. As it can be seen in figure $3 \mathrm{~d}$, this has also a beneficial effect for the fabrication of interdigitated finger structures for transistors.

Bulging effects which can typically occur on inkjet printed structures, ${ }^{[17,46]}$ can locally change the channel geometry and in worse case lead to a shortcut and therefore to a non- 
functional transistor. These effects can be actively suppressed when using pre-structured substrates. At this point it has to be annotated that the bulging of the electrode in figure $3 \mathrm{c}$ results from deviations in the opening of the mask (see figure S4 in the supporting information). As it can be seen in figure $3 \mathrm{e}$ the spreading and merging of the ink can be actively suppressed when using structured SAMs so that features separated by $<25 \mu \mathrm{m}$ can be processed in a simplified single printing step process. Furthermore, the developed process can be used independent of the ink. As it can be seen in figure 3f, similar result can be achieved by utilizing the water-based $\mathrm{Au}$-ink. To the best of our knowledge, transistors with such channel lengths have not been fabricated by industrial relevant inkjet printing in a single pass printing process or without the use of other beneficial effects as the coffee stain or the presence of physical barriers. ${ }^{[2,27]}$ These were the smallest feature sizes we could achieve with laser-cut masks. The smallest feature size that we achieved is already lower than the single drop diameter of the corresponding ink. In future experiments we plan to utilize higher-resolution patterning and lower drop volumes to even lower the resolutions we could achieve so far.

\subsection{Fully solution processable transistors}

Finally, we fabricated fully solution-processed transistors to investigate if the additional siloxane treatment has an influence on the device performance. With the developed process, we were able to replicate transistor structures, which are commonly fabricated by lithography and evaporation techniques. The bottom-source-drain electrodes are inkjetprinted as described above. As it can be seen in figure S6 in the supporting information, the silver electrodes showed a typical coffee-stain effect which resulted in a height of $76.31 \pm 10.8 \mathrm{~nm}$. The gold electrodes have a height of $128.48 \pm 5.7 \mathrm{~nm}$. Due to the low SFE of the SAM, which is still present in the transistor channel we were initially not able to inkjet print the semiconductor after the fabrication of the electrodes. Therefore, we removed the SAM with a 15 s Argon plasma treatment. This process was optimized in such way that it does not affect the conductivity of the electrodes but ensure a successful removal of the SAM. As shown in Table S1 of the SI, the Ar-plasma has no effect on the conductivity of the electrodes as the resistivity remains almost identical with $25.1 \pm 2.6 \mu \Omega \mathrm{cm}$ for the neat silver electrodes and $23.2 \pm 3.4 \mu \Omega \mathrm{cm}$ after the exposure to $15 \mathrm{~s}$ of Ar-plasma.

The fabrication process for the bottom-contact-top-gate transistors is schematically displayed in figure 4a. With this approach we obtained fully solution-processable transistors, with Ag or Au source- and drain-electrodes. As it can be seen in the detailed image (figure $4 \mathrm{~b}$ ) the process can be replicated on substrate areas $>25 \mathrm{~cm}^{2}$.

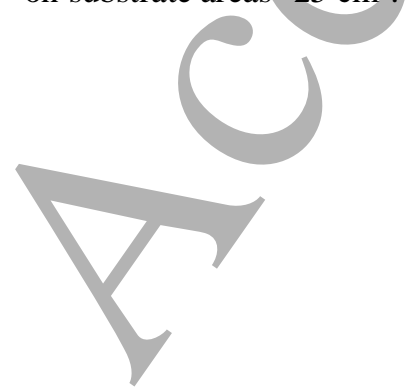

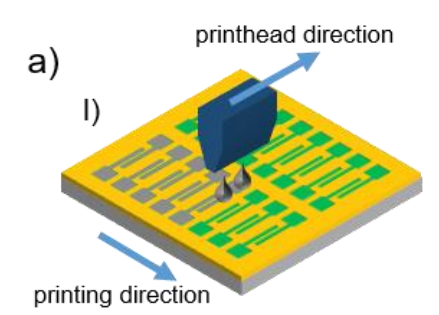
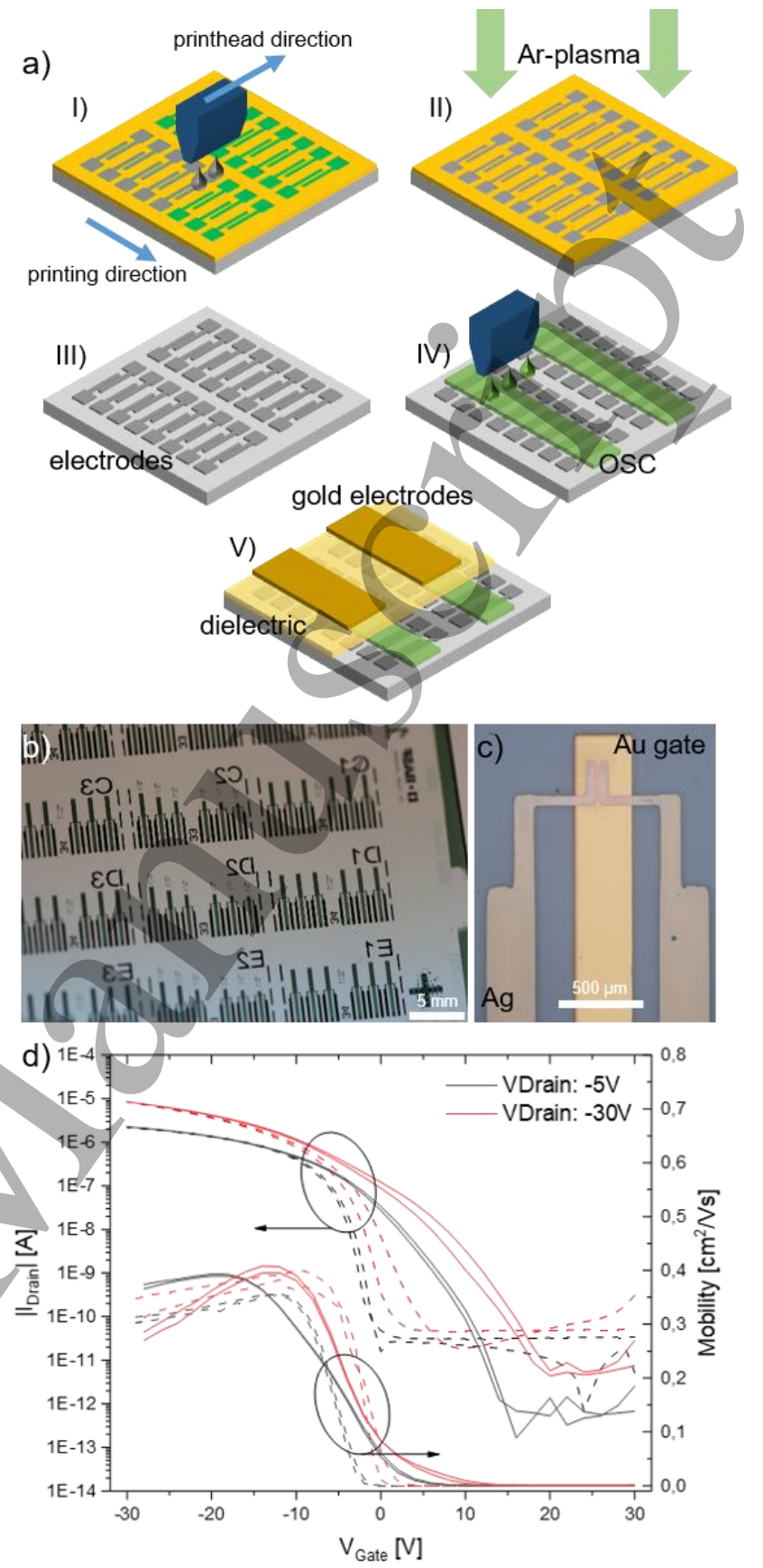

Figure 4: a) Schematic process of the transistor fabrication on pre-structured SAM substrates. b) Photograph of the fully solution processable transistors (white bar represents $5 \mathrm{~mm}$ ) and c) microscopic image of transistor with $25 \mu \mathrm{m}$ channel length. d) Transfer-characteristics of the best performing inkjet printed transistors (solid line) and reference devices (dashed line) in linear (black) and saturation (red) regime.

We obtained transistors with a channel length of down to $25 \mu \mathrm{m}$ (figure $4 \mathrm{c}$ ). The obtained transistors were electrically tested by determining the output- and transfer characteristics. For all the transistors the output-characteristics show ohmic behavior (see Figure S5 in the supporting information), thus a negative influence of the SAM and plasma treatment can be excluded. 
Furthermore, we compared the transfer characteristics to transistors fabricated by conventional lithography techniques (see table 4). Despite the fact that the printed electrodes show higher resistivity (silver: $2.5 \cdot 10^{-7} \Omega \mathrm{m}$; gold: $1.0 \cdot 10^{-6} \Omega \mathrm{m}$ ) compared to the bulk material (silver $1.6 \cdot 10^{-8} \Omega \mathrm{m}$; gold $\left.2.4 \cdot 10^{-8} \Omega \mathrm{m}\right)^{[47]}$, their overall device performance is comparable (see figure 4d). The extracted device characteristics averaged over at least 7 devices are displayed in table 3 . The transistors comprising of the printed electrodes show slightly lower linear (lin.) and saturated (sat.) mobility and higher on/off-current ratios. This can be attributed to the higher resistivity of the printed electrodes. Nonetheless, the developed process results in functional devices and the SAM structuring has no influence on the electrical performance.

Table 3: Comparison of the device characteristics of transistors with printed electrodes and the reference device.

\begin{tabular}{|l|c|c|c|c|c|c|}
\hline & \multicolumn{2}{|c|}{$\boldsymbol{\mu} \pm \boldsymbol{\sigma}\left[\mathrm{cm}^{\mathbf{2}} \mathbf{V s}^{-1}\right]$} & \multicolumn{2}{c|}{ On/off } & \multicolumn{2}{c|}{$\mathbf{V}_{\text {Th }}[\mathbf{V}]$} \\
& Lin. & Sat. & lin. & Sat. & Lin. & Sat. \\
\hline Printed $\mathrm{Ag}$ & $0.37 \pm 0.02$ & $0.39 \pm 0.03$ & $10^{5}$ & $10^{5}$ & -2.12 & 2.07 \\
\hline Printed $\mathrm{Au}$ & $0.25 \pm 0.01$ & $0.10 \pm 0.01$ & $5 \cdot 10^{4}$ & $10^{5}$ & 2.92 & 1.55 \\
\hline $\begin{array}{l}\text { Evaporated } \\
\mathrm{Au}\end{array}$ & $0.39 \pm 0.01$ & $0.45 \pm 0.02$ & $10^{6}$ & $10^{6}$ & -4.85 & -0.18 \\
\hline
\end{tabular}

\section{Conclusion}

Although digital printing techniques, like inkjet printing, offer the possibility to fabricate separate structures, they mostly suffer from a low lateral resolution or the necessity to optimize the ink composition or print patterns. Introducing a surface energy patterning based on a low energetic siloxane SAM facilitates the solution processing of arbitrary structures by generating an anisotropic energetic landscape, which controls the flow and spreading of the deposited ink. Due to the confined flow of liquid we were able to increase the lateral resolution and achieve feature sizes $<25 \mu \mathrm{m}$ in a single step printing process, replicating shadow masks typically used for thermal evaporation. The developed process is independent of the fluid physical properties of the ink, which is especially beneficial when using commercially available inkjet inks, whose ink composition should not be altered. The combination of such a pre-structured 2D surface with inkjet technology is of particular importance as it allows fabrication of arbitrary shapes without having to take into account the negative effects resulting from pattern design, deposition parameters or the substrate-ink-interaction. Despite the fact that the described process limits the advantages of inkjet printing, namely the mask-free production of different patterns, it benefits from an improved resolution. The presented approach helps to facilitate the fabrication of highresolution patterns without the need of elaborated vacuum deposition techniques and with reduced materials consumption. Combining inkjet printing with a digital structuring process the presented approach can be used to fabricate highly customizable patterns. Although in this approach we carry out an additional structuring process compared to typical printing processes, it allows the fabrication of features below the native resolution resulting from the substrate-ink-interaction on the given substrate without the need of conventional evaporation techniques.

By fabricating fully functional OFETs on such surface energy patterned substrates, we have shown that the additional prestructuring steps do not affect the device performance. Since the formation of siloxane based SAMs is not bound to a specific substrate, once developed, this process can be easily transferred to other ink systems, substrates or layouts without the need of print parameter or ink optimization resulting in a reduced process development time.

\section{Acknowledgements}

We thank Martin Held and Tobias Rödlmeier for fruitful discussion. This research has been funded by the Deutsche Forschungsgemeinschaft (DFG, German Research Foundation) under Germany's Excellence Strategy via the Excellence Cluster 3D Matter Made to Order (EXC-2082/1390761711), by the German Federal Ministry for Research and Education (BMBF) through the project FKZ: 03INT606AG.References

[1] I. Salaoru, S. Maswoud, S. Paul, Micromachines 2019, 10, 417.

[2] S. Khan, L. Lorenzelli, R. S. Dahiya, IEEE Sens. J. 2015, 15, 3164.

[3] L. Inzelberg, M. David Pur, S. Schlisske, T. Rödlmeier, O. Granoviter, D. Rand, S. Steinberg, G. Hernandez-Sosa, Y. Hanein, Flex. Print. Electron. 2018, 3, 045001.

M. Mayer, A. J. Baeumner, Chem. Rev. 2019, 119, 7996.

[5] C. Vega-Colado, B. Arredondo, J. Torres, E. LópezFraguas, R. Vergaz, D. Martín-Martín, G. del Pozo, B. Romero, P. Apilo, X. Quintana, M. A. Geday, C. de Dios, J. Sánchez-Pena, Sensors 2018, 18, 3045.

[6] N. Strobel, N. Droseros, W. Köntges, M. Seiberlich, M. Pietsch, S. Schlisske, F. Lindheimer, R. R. Schröder, U. Lemmer, M. Pfannmöller, N. Banerji, G. Hernandez-Sosa, Adv. Mater. 2020, 32, 1908258.

[7] P. Feng, H. Ji, L. Zhang, X. Luo, X. Leng, P. He, H. Feng, J. Zhang, X. Ma, W. Zhao, Nanotechnology 2019, 30, 185501.

[8] T. M. Eggenhuisen, Y. Galagan, A. F. K. V. Biezemans, T. M. W. L. Slaats, W. P. Voorthuijzen, S. Kommeren, S. Shanmugam, J. P. Teunissen, A. Hadipour, W. J. H. Verhees, S. C. Veenstra, M. J. J. Coenen, J. Gilot, R. Andriessen, W. A. Groen, $J$. Mater. Chem. A 2015, 3, 7255.

[9] D. Corzo, K. Almasabi, E. Bihar, S. Macphee, D. Rosas-Villalva, N. Gasparini, S. Inal, D. Baran, $A d v$. Mater. Technol. 2019, 4, 1900040.

[10] G. Cummins, M. P. Y. Desmulliez, Circuit World 
2012, 38, 193.

[11] A. Sandström, L. Edman, Energy Technol. 2015, 3, 329.

[12] G. Dell'Erba, A. Perinot, A. Grimoldi, D. Natali, M. Caironi, Semicond. Sci. Technol. 2015, 30, 104005.

[13] A. Bucciarelli, C. R. Chandraiahgari, A. Adami, V. Mulloni, L. Lorenzelli, Flex. Print. Electron. 2020 , DOI 10.1088/2058-8585/abbb7e.

[14] M. Kuang, L. Wang, Y. Song, Adv. Mater. 2014, 26, 6950.

[15] B.-J. de Gans, U. S. Schubert, Langmuir 2004, 20, 7789.

[16] FUJIFILM Dimatix Inc., 2015, 2.

[17] B. Derby, Annu. Rev. Mater. Res. 2010, 40, 395.

[18] J. H. Oh, S. Y. Lim, J. Micromech. Microeng. 2010 , 20, 015030 .

[19] T. Schmaltz, G. Sforazzini, T. Reichert, H. Frauenrath, Adv. Mater. 2017, 29, 1605286.

[20] S. P. Pujari, L. Scheres, A. T. M. Marcelis, H. Zuilhof, Angew. Chemie - Int. Ed. 2014, 53, 6322.

[21] S. Schlisske, M. Held, T. Rödlmeier, S. Menghi, K. Fuchs, M. Ruscello, A. J. Morfa, U. Lemmer, G. Hernandez-Sosa, Langmuir 2018, 34, 5964.

[22] B. J. Kang, J. H. Oh, Surf. Coatings Technol. 2010, 205, S158.

[23] B. J. Kang, Y. S. Kim, Y. W. Cho, J. H. Oh, Microelectron. Eng. 2011, 88, 2355.

[24] R. J. Good, J. Adhes. Sci. Technol. 1992, 6, 1269.

[25] J. Z. Wang, Z. H. Zheng, H. W. Li, W. T. S. Huck, H. Sirringhaus, Nat. Mater. 2004, 3, 171.

[26] R. Eckstein, M. Alt, T. Rödlmeier, P. Scharfer, U. Lemmer, G. Hernandez-Sosa, Adv. Mater. 2016, 28, 7708.

[27] C. Bali, Coffee-Ring-Effect Based Self-Assembly Mechanism for All-Inkjet Printed Organic Field Effect Transistors with Micron-Sized Channel Length, TU Chemnitz, 2020.

[28] H. Sirringhaus, T. Kawase, R. H. Friend, T. Shimoda, M. Inbasekaran, W. Wu, E. P. Woo, Science 2000, 290, 2123.

[29] W.-T. Park, Y.-Y. Noh, J. Mater. Chem. C 2017, 5, 6467.

[30] A. Pierre, M. Sadeghi, M. M. Payne, A. Facchetti, J. E. Anthony, A. C. Arias, Adv. Mater. 2014, 26, 5722.

[31] S. Kang, B.-Y. Lee, S.-H. Lee, S.-D. Lee, Surface Tension in Microsystems, Springer Berlin Heidelberg, Berlin, Heidelberg, 2013.

[32] W. J. Hyun, E. B. Secor, F. Zare Bidoky, S. B. Walker, J. A. Lewis, M. C. Hersam, L. F. Francis, C. D. Frisbie, Flex. Print. Electron. 2018, 3, 035004.

[33] M. D. Mamo, E. S. Shin, Y. Y. Noh, J. Mater. Chem. C 2017, 5, 10900.

[34] C. McDowell, M. Abdelsamie, M. F. Toney, G. C. Bazan, Adv. Mater. 2018, 30, 1707114.

[35] D. Jang, D. Kim, J. Moon, Langmuir 2009, 25, 2629.

[36] J. C. Bijleveld, A. P. Zoombelt, S. G. J. Mathijssen, M. M. Wienk, M. Turbiez, D. M. De Leeuw, R. A. J. Janssen, J. Am. Chem. Soc. 2009, 131, 16616.
[37] L. Shaw, H. Yan, X. Gu, P. Hayoz, R. T. Weitz, D. Kaelblein, M. F. Toney, Z. Bao, Macromolecules 2018, 51, 4325.

[38] L. Shaw, P. Hayoz, Y. Diao, J. A. Reinspach, J. W. F. To, M. F. Toney, R. T. Weitz, Z. Bao, ACS Appl. Mater. Interfaces 2016, 8, 9285.

[39] D. K. Owens, R. C. Wendt, J. Appl. Polym. Sci. 1969, 13, 1741.

[40] D. H. Kaelble, J. Adhes. 1970, 2, 66.

[41] T. Young, Philos. Trans. R. Soc. London 1805, 95 , 65.

[42] P. Lambert, Surface Tension in Microsystems: Engineering Below the Capillary Length, Springer Berlin Heidelberg, 2013.

[43] G. Grau, J. Cen, H. Kang, R. Kitsomboonloha, W. J. Scheideler, V. Subramanian, Flex. Print. Electron. 2016, 1, 023002.

[44] Süss MicroTec SE, 2020.

[45] R. Abbel, P. Teunissen, J. Michels, W. A. Groen, Adv. Eng. Mater. 2015, 17, 615.

[46] J.-S. Kwon, D. Lee, J. Oh, Appl. Sci. 2018, 8, 280.

[47] R. A. Matula, J. Phys. Chem. Ref. Data 1979, 8, 1147. 